bioRxiv preprint doi: https://doi.org/10.1101/2020.04.03.022905; this version posted January 22, 2021. The copyright holder for this preprint

(which was not certified by peer review) is the author/funder, who has granted bioRxiv a license to display the preprint in perpetuity. It is made available under aCC-BY-NC-ND 4.0 International license.

\title{
Size-dependent eco-evolutionary feedbacks in fisheries
}

Eric Edeline ${ }^{1,2 *}$, Nicolas Loeuille ${ }^{1}$

1: Sorbonne Université/UPMC Univ. Paris 06/CNRS/INRA/IRD/Paris Diderot Univ. Paris 07/UPEC/

5 Institut d'Ecologie et des Sciences de l'Environnement - Paris (iEES-Paris), 4 Place Jussieu, FR-75252

Paris, France.

2: ESE Ecology and Ecosystem Health, INRAE, Agocampus Ouest, 35042 Rennes, France.

* Corresponding author: eric.edeline@inrae.fr 
Size-dependent eco-evo feedback loops

\section{Abstract}

Harvesting may drive body downsizing along with population declines and decreased harvesting yields.

These changes are commonly construed as direct consequences of harvest selection, where small-

15 bodied, early-reproducing individuals are immediately favoured. However, together with directly selecting against a large body size, harvesting and body downsizing alter many ecological features, such as competitive and trophic interactions, and thus also indirectly alter natural selection acting back on body sizes through eco-evolutionary feedback loops (EEFLs). We start this essay by reviewing the conditions under which natural selection favours either larger or smaller body sizes. Then, we analyse

20 simple EEFLs in which one-dimensional density-dependent natural selection acts either antagonistically or synergistically with direct harvest selection on body size. Antagonistic feedbacks favour body-size stasis but erode genetic variability and associated body-size evolvability, and may ultimately impair population persistence and recovery. In contrast, synergistic feedbacks drive fast evolution towards smaller body sizes and favour population resilience, but may have far-reaching

25 bottom-up or top-down effects. We illustrate the further complexities resulting from multiple environmental feedbacks using a co-evolving predator-prey pair. In this case, outcomes from EEFLs depend not only on densities, but also on whether prey sit above or below the optimal predator/prey body-size ratio, and whether prey have a higher or lower body-size evolvability than predators. EEFLs challenge our ability to predict population and trait dynamics and, to be understood, will require

30 investigation programs documenting natural selection and its response to phenotypic and environmental changes.

Key words: Body size, Co-evolution, Competition, Density-dependent selection, Eco-evolutionary feedbacks, Harvesting, Natural selection, Predation. 


\section{Glossary}

Absolute fitness: number of offspring reaching the reproductive stage.

Evolutionary deterioration: evolutionary change leading to smaller population densities, thereby increasing its probability of extinction (e.g., due to demographic stochasticity).

Evolutionary rescue: adaptive evolutionary change that restores positive growth to declining

populations and prevents extinction.

Evolvability: trait potential to evolve.

Fitness landscape: multidimensional surface depicting fitness as a function of phenotypic traits.

Relative fitness: absolute fitness of a given phenotype divided by average absolute fitness of all phenotypes in the population.

45 Selection gradient: Trait-specific slope of the fitness landscape, i.e., holding other traits constant.

\section{Introduction}

The management of exploited populations is classically based on density-dependent population models in which harvesting, while decreasing population size, also relaxes density-dependent competition so

50 that individual biomass productivity is increased (Verhulst 1838, Schaefer 1954, Hilborn and Walters 1992). However, this classical view has been repeatedly challenged by studies showing that individual biomass productivity often tends to decrease, not to increase, with harvesting (Kristiansen and Svåsand 1998, Conover and Munch 2002, Swain et al. 2007, Edeline et al. 2007, 2009, Biro and Post 2008, Heino et al. 2013). 


\section{Size-dependent eco-evo feedback loops}

This negative relationship between harvest effort and individual biomass production is generally interpreted as a rapid evolutionary response to direct harvest selection against large-bodied individuals by fishers (Trippel 1995, Law 2000, Kuparinen and Merilä 2007, Fenberg and Roy 2008, Heino et al. 2015). Accordingly, selection against a large body size is expected to favour slow-growing and early-

60 maturing genotypes, which also tend to have lower fecundity and decreased offspring quality (Walsh et al. 2006, Heino et al. 2013). However, cases remain where exploitation induces no phenotypic change (Hilborn and Minte-Vera 2008, Devine and Heino 2011, Silva et al. 2013, Marty et al. 2014), or a change towards larger body sizes as predicted by density-dependent population models (Hilborn and Minte-Vera 2008). Therefore, whether harvest-induced evolutionary changes occur at all, or are large and rapid enough to influence biomass productivity remains controversial (Andersen and Brander 2009, Borrell 2013).

This debate, we feel, is plagued by a pervasive inclination of many researchers to overlook natural selection and to consider selective removal by harvesters as the only dynamic selective force at play.

70 Natural selection, if ever mentioned, is regarded as negligible such that harvest-induced changes are widely considered as slowly reversible (see e.g. the seminal paper by Law 2000). The simplifying assumption that natural selection is negligible further leads to conclude, for instance, that body-size stasis in harvested populations indicates that evolution is absent or has unimportant effects relative to the effects of ecology (e.g., Hilborn and Minte-Vera 2008). As we propose below, body-size stasis may

75 in fact reflect eco-evolutionary dynamics in which natural selection opposes the effects of direct harvest selection. Failure to account for these eco-evolutionary processes might fundamentally hamper our ability to understand and, hence, to manage productivity dynamics in harvested populations. 


\section{Size-dependent eco-evo feedback loops}

The objective of this assay is to provide an impetus to the study of natural selection in harvested

80 populations, and may be seen as a complementary follow-up to Kinnison et al. (2015). Reviewing theoretical, experimental and empirical insights, we elaborate plausible scenarios under which natural selection dynamically acts in parallel with direct harvest selection to affect the yield and resilience of harvested populations. We first review the mechanisms through which natural selection may favour either large-bodied or small-bodied individuals. Secondly, we build on this knowledge of natural

85 selection to identify the different pathways and directions that size-dependent EEFLs may take in harvested populations when only one single species evolves. In the third section, we extend the approach to EEFLs acting at the two-species and food-web levels. Finally, in the fourth section we conclude with an overview of the methods currently available to advance our empirical knowledge of EEFLs and with a consideration of how EEFLs may change our approach to managing harvested 90 populations.

\section{Size-dependent natural selection}

Ample evidence shows that trait evolution in response to natural selection may be large and fast, hence

95 far from negligible (Grant and Grant 2002, Stockwell et al. 2003, Hairston et al. 2005, Carroll et al. 2007). This section provides details of the mechanisms through which natural selection moulds body sizes, but readers interested only in the results may refer to Table 1 and move directly to Section 2 . 
Size-dependent eco-evo feedback loops

Table 1. Sources of natural selection predicted and observed to favour either a smaller or larger body size at age or at maturity.

\begin{tabular}{|c|c|c|c|}
\hline $\begin{array}{l}\text { Natural selection for a } \\
\text { Smaller body size }\end{array}$ & \multicolumn{3}{|c|}{$\begin{array}{l}\text { Natural selection for a } \\
\text { Larger body size }\end{array}$} \\
\hline Exploitative competition ${ }^{1}$ & $\begin{array}{l}\text { (De Roos et al. 2003, } \\
\text { Kooijman 2010) }\end{array}$ & Interference competition & $\begin{array}{l}\text { (Le Bourlot et al. } \\
\text { 2014) }\end{array}$ \\
\hline Long-term food stress ${ }^{2}$ & (Arendt 1997) & Long-term food stress ${ }^{2}$ & $\begin{array}{l}\text { (Gadgil and Bossert } \\
\text { 1970) }\end{array}$ \\
\hline $\begin{array}{l}\text { Selective predation on large-bodied } \\
\text { individuals in prey populations }\end{array}$ & $\begin{array}{l}\text { (Gårdmark and } \\
\text { Dieckmann 2006, } \\
\text { Heino et al. 2015) }\end{array}$ & Seasonal food stress & $\begin{array}{l}\text { (van de Wolfshaar et } \\
\text { al. 2008) }\end{array}$ \\
\hline Size-independent predation & $\begin{array}{l}\text { (Abrams and Rowe } \\
\text { 1996, Gårdmark and } \\
\text { Dieckmann 2006, } \\
\text { Heino et al. 2015) }\end{array}$ & $\begin{array}{l}\text { Selective predation on small- } \\
\text { bodied individuals in prey } \\
\text { populations }{ }^{3}\end{array}$ & (Day et al. 2002) \\
\hline \multirow[t]{2}{*}{$\begin{array}{l}\text { Selective predation on juvenile (immature) } \\
\text { individuals in prey populations }\end{array}$} & $\begin{array}{l}\text { (Abrams and Rowe } \\
\text { 1996) }\end{array}$ & Cannibalism & $\begin{array}{l}\text { (Claessen et al. } \\
\text { 2004) }\end{array}$ \\
\hline & & $\begin{array}{l}\text { Selective predation on mature } \\
\text { individuals in prey populations }\end{array}$ & $\begin{array}{l}\text { (Ernande et al. 2004, } \\
\text { Heino et al. 2015) }\end{array}$ \\
\hline
\end{tabular}

105 1: Note, however, that selection may be for a larger (not smaller) body size if attack rate increases faster with body size than maintenance metabolic rate. See text for more details. 2: long-term food stress has opposite effects on size-at-age and on size-at-maturity. 3: direction of selection may be different on size-at-age and on size-at-maturity, see text.

Competition may be exploitative, i.e., resource-mediated (or indirect) or interference-mediated, i.e., direct. Both types of competition are expected to generate selection on body size, but only exploitative competition is expected to favour smaller body sizes. Exploitative competition may be usefully construed using the $R^{*}$ rule, which states that competition selects individuals surviving on the lowest

115 equilibrium resource level (Tilman 1982). A lower individual $R^{*}$ (i.e., a higher competitive ability) is achieved by increasing resource intake and/or by decreasing basal metabolic requirements. Note, however, that both resource intake and basal metabolic rate generally increase with body size (Peters 1983, Persson et al. 1998, De Roos et al. 2003, Kooijman 2010). Hence, whether individual $R^{*}$ increases or decreases with body size depends on the relative strengths of allometric constraints acting 


\section{Size-dependent eco-evo feedback loops}

120 on resource intake and metabolic rate. If resource intake increases faster with body size than metabolic rate, $R^{*}$ decreases with increasing body size and exploitative competition should select for larger body sizes. In contrast, if resource intake increases slower than metabolic rate, $R^{*}$ increases with body size and exploitative competition should select for smaller body sizes. In fish, available evidence suggests that $R^{*}$ increases with body size (Persson and De Roos 2006), so that exploitative competition should

125 favour smaller sizes. The argument extends to many other taxa if one assumes that ingestion increases with body surface ( $\propto$ size $^{2}$ ) while maintenance increases with body volume $\left(\propto\right.$ size $\left.^{3}\right)$ (Kooijman 2010). Population dynamics consistent with this prediction have been reported in the vendace Coregonus albula (Hamrin and Persson 1986), roach Rutilus rutilus (Persson et al. 1998) and Japanese medaka Oryzias latipes (Edeline et al. 2016).

Competition, if not leading to competitive exclusion, may also select on body sizes indirectly through decreasing the individual resource share on the long term. Available evidence suggests that such food stress has opposite effects on somatic growth rate and age at maturation. Across a wide variety of taxa, food stress favours slower growth rates and smaller size at age, presumably by imposing energy

135 reallocation to the most vital functions (Arendt 1997). In contrast, fitness-maximising models predict that food stress should select for delayed maturation and, hence, for larger size at maturity if somatic growth rate is constant (Gadgil and Bossert 1970), a prediction supported by available empirical evidence (Holliday 1989, Sgrò and Partridge 2000). Therefore, if somatic growth and maturation trade off, the growth-mediated and maturation-mediated effects of food stress on body size oppose each 140 other, and are thus likely to remain inconsistent or cryptic. 
Competition is not the only ecological interaction that may select for smaller body sizes. Predators that target large-bodied prey directly select for smaller prey body sizes just like harvesters do (see above). This is for instance the case for fish predation on zooplankton (Brooks and Dodson 1965). If predators

145 are non size-selective, predators still favour earlier maturation in prey and, hence, a smaller size at maturity if somatic growth is constant (Abrams and Rowe 1996). This is because early-maturing individuals have an increased fitness advantage when life expectancy is reduced (Gårdmark and Dieckmann 2006, Heino et al. 2015). Finally, if predation mortality is stage-dependent, higher juvenile (immature) mortality favours earlier maturity which, given a fixed somatic growth rate, also means 150 maturity at a smaller body size (Abrams and Rowe 1996, Heino et al. 2015).

\subsection{Natural selection for a larger body size}

In the wild, survival often increases with larger body sizes (Roff 1992), as demonstrated for instance in juvenile fish (Perez and Munch 2010, Stige et al. 2019), juvenile Soay sheep (Ovis aries, Hunter et al.

155 2018), or adult fish (e.g., Carlson et al. 2007, Olsen and Moland 2011). The mechanism behind this positive survival-size relationship could involve a higher resistance to starvation in larger-bodied individuals (van de Wolfshaar et al. 2008), but also results from strong interference in competitive interactions. While size-selective effects of exploitative competition are dependent upon the allometric scaling exponents of intake and maintenance rates (see above), interference competition almost

160 universally brings an advantage to large-sized individuals in contests for food (Persson 1985, Post et al. 1999). In fish, this phenomenon is so prevalent that aquaculturists must apply size culling to fish cohorts to prevent a few giant individuals to monopolize food, even though food is provided in excess. In experimental populations of the springtail Folsomia candida, interference favours large-sized 
individuals that can monopolize resources (Le Bourlot et al. 2014). Similarly, in wild populations of the

165 brown anole lizard Anolis sagrei natural selection for larger body sizes increases in parallel with population density and associated interference competition (Calsbeek and Smith 2007). In these lizards, the strength of competition-induced selection on body size overwhelmed the strength of predationinduced selection (Calsbeek and Cox 2010).

170 Often, predators are size-limited and thus preferentially feed on small-sized prey individuals. This is true not only in aquatic, but also in terrestrial systems (Sinclair et al. 2003). In such cases, predators favour prey individuals that grow fast through a "predation window" to rapidly reach a size refuge, i.e., they select for a large body size at a given age (Day et al. 2002). This predation window plays a key role in mediating the population dynamic effect of intraspecific predation (i.e., cannibalism), an

175 interaction that is present in multiple aquatic or terrestrial taxa (Fox 1975, Claessen et al. 2002, 2004). Cannibalism is presumably the mechanism that controlled the positive effect of population density on somatic growth rate in the Windermere pike population where, as the density of cannibals increased, survival was biased towards faster-growing individuals (Edeline et al. 2007, 2009).

180 The effect of size-limited predation on age at maturation is less straightforward than on somatic growth. If mortality increases among small-sized individuals, predictions depend on the details of the model. Optimality models predict evolution of delayed maturation at a larger body size (Taylor and Gabriel 1992). In contrast, adaptive dynamics models accounting for a trade off between somatic growth and reproduction and for a positive effect of body size on fecundity lead to more complex 185 outcomes: increased mortality among small-sized individuals can increase or decrease maturation size, or even lead to the coexistence of both early- and late-maturing individuals when benefits from early 
190 more often supports delayed maturation at a larger body size when predation targets small-sized individuals (Edley and Law 1988, Wellborn 1994, Beckerman et al. 2010, Le Rouzic et al. 2020).

Finally, a larger body size further provides females with a higher fecundity in egg-spawning species (Barneche et al. 2018), and males with a strong advantage in contest sexual selection (e.g., Fleming and

195 Gross 1994). Combined together, these multiple positive effects of natural selection on body sizes are likely to outweigh the negative effects, as suggested by an overall tendency for natural and sexual selection to favour larger body sizes across multiple taxa (Kingsolver and Pfennig 2004). We now move to examining how such size-dependent natural selection may interact dynamically with ecology in EEFLs.

\section{Theory and scenarios of harvest-induced EEFLs with one evolving species}

\section{Box1. Defining the selection- and evolvability-mediated pathways to eco-evolutionary feedback loops (EFFLs).}

To study existing feedbacks between ecological and evolutionary dynamics, two main frameworks are currently used: quantitative genetics (QG) and adaptive dynamics (AD). Though the two methods differ, they are both based on the idea that the description of trait dynamics in response to selection requires two fundamental ingredients: trait(s) evolutionary potential (hereafter “evolvability”) and a measure of selection acting on the trait(s) (Abrams 2001). 
Size-dependent eco-evo feedback loops

Consider for instance the classical recursive equation of quantitative genetics (QG):

$$
\bar{x}_{t+1}=\bar{x}_{t}+\Delta \bar{x}=\bar{x}_{t}+\underbrace{\operatorname{Va} a_{x}}_{\text {Evolvability }} \frac{\operatorname{cov}(w, x)}{\operatorname{var}(x)} \quad \text { Eq. 1, }
$$

where $\bar{x}$ is the mean population value of a univariate trait $x, t$ is generation index, $V a_{x}$ is

additive genetic variance, $\quad w$ is relative individual fitness, and $\frac{\operatorname{cov}(w, x)}{\operatorname{var}(x)}$ is the directional selection gradient, i.e., the slope of the linear regression between relative fitness and trait $x$ (Lande and Arnold 1983). Provided that the definition of $w$ includes at least density dependence and/or frequency dependence, Eq. 1 incorporates selection-mediated EEFLs as the ecological context (density or frequency) then impacts the selection term (Abrams 2001). Eco-evolutionary feedback loops may also occur through the evolvability-mediated pathway in Eq. 1, for instance if $V a_{x}$ is directly linked to the demographic context (e.g., an existing correlation between population density and genetic variability) or if $V a_{x}$ is an explicit function of the strength of selection since strong directional selection is expected to decrease additive genetic variances (Crow 2008).

Adaptive dynamics (AD) (Dieckmann and Law 1996) readily account for both selection- and evolvability-mediated EEFLs. This essential feature of $\mathrm{AD}$ is captured by the canonical equation: 


$$
\frac{d \bar{x}}{d t}=\left.\underbrace{\frac{1}{2} \mu \sigma^{2} N^{*}(x)}_{\text {Evolvability }} \underbrace{\frac{\partial W\left(x^{\prime}, x\right)}{\partial x^{\prime}}}_{\text {Selection }}\right|_{x^{\prime}=x} \quad \text { Eq. 2, }
$$

where $x$ is a resident trait, $x^{\prime}$ is a mutant trait, $d \bar{x} / d t$ is a continuous-time analogue of $\Delta \bar{x}$ in Eq. $1, \mu$ is per capita mutation rate, and $\sigma^{2}$ is phenotypic variance from a mutation. $N^{*}(x)$ is equilibrium population size for the resident trait, and Eq. 2 hence incorporates the evolvabilitymediated pathway to EEFLs since evolvability, here determined by the mutation process, is explicitly dependent on equilibrium population size $N^{*}(x)$, which is set by the value of the resident trait $x$ $W\left(x^{\prime}, x\right)$ is invasion fitness for a mutant trait $x^{\prime}$ in an environment determined by the resident trait $x$. Because this fitness definition is based on ecological dynamics, one sees that selectionmediated EEFLs are readily considered in adaptive dynamics models. Finally $\left(\partial W\left(x^{\prime}, x\right)\right) /\left(\partial x^{\prime}\right)$ is the directional selection gradient acting on the mutant trait $x^{\prime}$, i.e., is the invasion criterion (slope of the fitness landscape for $x^{\prime}$ evaluated in $x$ ).

Theory presented in Box 1 predicts that EEFLs may proceed through two different pathways: a selection-mediated and an evolvability-mediated pathways, which we illustrate in Fig. 1. The selectionmediated pathway is captured by Arrow 1 (Fig. 1): the environment of an individual generates natural selection on body size (see Section 1 above). In addition to influencing individual fitness and, from

245 there, population densities, body size has widespread and consistent ecological effects (Peters 1983, Brown et al. 2004, Woodward et al. 2005). Hence, selection-induced change in body size, in turn, may impact the environment through the size-dependency of reproductive success and ecological interactions (Arrow 2). The evolvability-mediated pathway to size-dependent EEFLs is captured by 
Arrow 3, and involves mutation-limitation effects linked to population sizes and/or any other existing

correlations between genetic diversity and population size (Box 1). Such evolvability-mediated pathways to EEFLs are often neglected, but may actually be important from a management or a conservation point of view (Carlson et al. 2014, Marty et al. 2015, Kuparinen and Hutchings 2017). Harvesting may trigger or disrupt size-dependent EEFLs through both direct harvest selection on body size and through the removal of conspecifics and possibly also heterospecifics (Fig. 1).

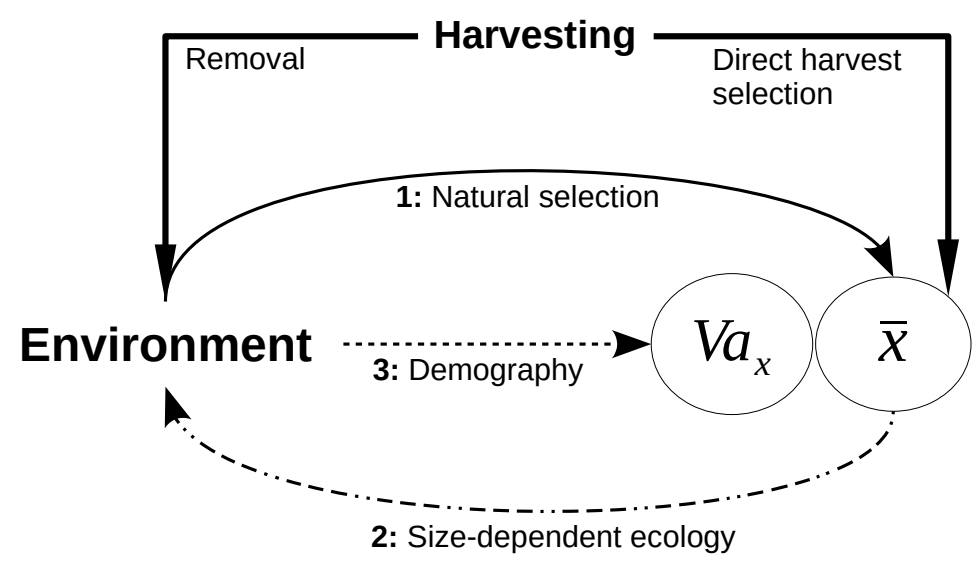

Figure 1: Selection-mediated and evolvability-mediated pathways to sizedependent eco-evolutionary feedback loops (EEFLs). $\quad V a_{x}$ and $\bar{x}$ stand for additive genetic variance and mean body size, respectively, in the harvested population (Box 1). Arrow 1: natural selection and the selection-mediated pathway to EEFLs, Arrow 2: body size-dependent ecological processes, Arrow 3: Effects of demography on genetic variability and the evolvability-mediated pathway to EEFLs (Box 1). NB 1: phenotypic plasticity, as also captured by Arrow 1, will not be discussed. NB 2: For the sake of simplicity, we did not consider the potential direct effects of selection on additive genetic variances (e.g. Crow 2008).

In order to fully grasp the basic ideas that underpin size-dependent EEFLs in the system depicted by Fig. 1, we provide a graphical representation of a moving adaptive landscape in Fig. 2. For simplicity, the fitness landscapes represented on fig 2 ignore frequency dependent selection, so that each

260 phenotype has a given fitness irrespective of its frequency. This fitness would be representative of the absolute fitness of the corresponding monomorphic population. Relative fitness of this phenotype confronted to another can then simply be read from the relative position on the fitness landscapes, phenotypes with higher fitness being selected. Therefore this representation, though it simplifies the 


\section{5 because population persistence depends on absolute, not relative fitnesses, and because absolute fitness} is therefore more intuitively linked with management aspects. For more discussion on the link between absolute and relative fitness, see Orr (2007).

Second, we represent what we believe are the most generic functional forms for absolute fitness

270 functions: harvest selection is often directional against a large body size, and body size is often constrained by trade-offs resulting in stabilizing natural selection (Kingsolver et al. 2001, Kingsolver and Pfennig 2004, Carlson et al. 2007). Note that this assumption of stabilizing natural selection also follows from the general observation that evolution towards smaller body sizes is associated with severe fitness costs (e.g., Walsh et al. 2006), while largest-bodied and oldest individuals may be subject

275 to senescence. Although these settings are very simple and maybe rare in nature, their evolutionary outcome is more easily visualized than when multiple environmental feedbacks operate simultaneously and prevent evolutionary optimization (Metz et al. 2008).

In the absence of any direct harvest selection, the population mean body size resides at the naturally-

280 selected body size optimum (dotted blue curve, $t_{0}$ in Fig. 2A). The product of natural selection with direct harvest selection (i.e., survival to harvesting, dashed red curve) instantaneously warps the naturally-selected fitness landscape to generate a new, composite fitness landscape (solid grey curve) on which the population mean trait value is associated with a fitness at which the population crosses the extinction threshold ( $\mathrm{t}_{1}$, maladaptation). Rapid adaptive evolution through a few generations towards 285 the newly-selected adaptive optimum restores a fitness at which the population may persist $\left(\mathrm{t}_{2}\right.$, readaptation). If adaptive change occurs fast enough, it may potentially restore a positive population 
Size-dependent eco-evo feedback loops

growth and prevent extinction, a process termed “evolutionary rescue” (Glossary, Gomulkiewicz and Holt 1995).

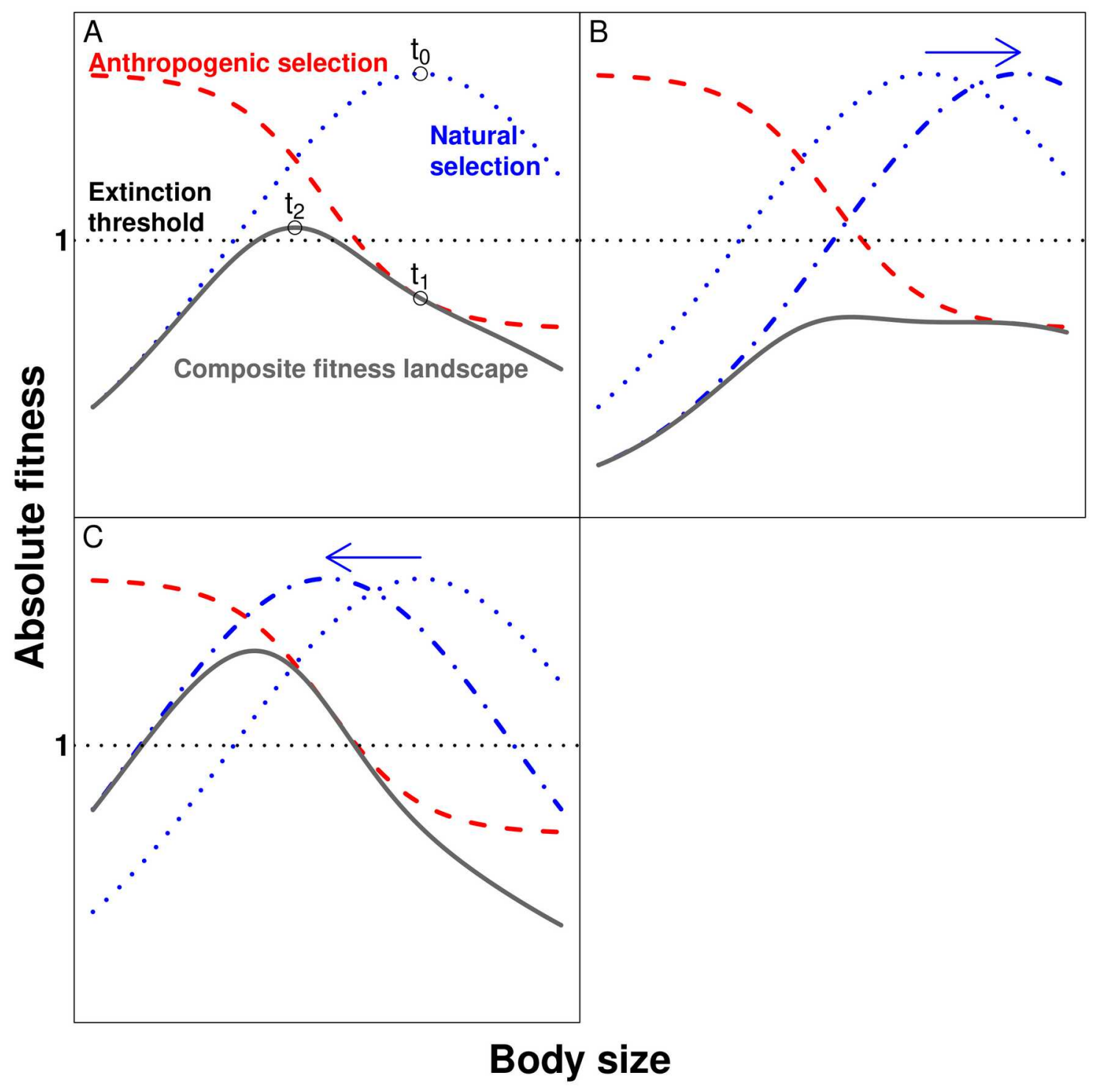

Figure 2: Eco-evolutionary feedbacks in harvested populations. Curves show the relationship between absolute fitness and body size, and the horizontal dotted line shows unity absolute fitness (extinction threshold). A: Evolutionary "rescue" (see Glossary) with no eco-evolutionary feedback loop (EEFL). Open circles show the mean phenotype in the population. B: A single density parameter feedbacks on natural selection, generating an antagonistic EEFL. The arrow shows the change in directional natural selection due to the environmental feedback. C: The one-dimensional density-dependent feedback generates a synergistic EEFL in which natural selection changes to reinforce the effect of direct harvest selection. 
290 The model presented in Fig. 2A makes the simplifying assumption that natural selection does not respond to harvesting. However, in addition to imposing direct harvest selection on body size, harvesting also alters the environment (Fig. 1, Arrow 1) and may thus indirectly change natural selection acting on body size (Bouffet-Halle et al. 2020). We will now examine two scenarios in which such harvest-induced changes in natural selection either oppose or reinforce the action of direct harvest 295 selection on body size.

\subsection{Antagonistic EEFLS}

We first consider a feedback in which harvesting changes natural selection towards favouring largerthan-initial body sizes (sketched in Fig. 2B). As this selection acts in opposite ways to the direct

300 selective effects of harvesting, we refer to this situation as an antagonistic EEFL. Compared to an EEFL-absent case, antagonistic EFFLs magnify warping of the adaptive landscape and thus impair population persistence. At an extreme, the fitness peak may dwindle below the extinction threshold (Fig. 2B). Antagonistic EEFLs are expected whenever density-dependent natural selection favours small body sizes and harvesting, through reducing densities, relaxes natural selection for a small body

305 size (Fig. 2B). For instance, reduced population densities may relax exploitative competition for resources, and weaken associated selection for smaller body sizes (Table 1). Antagonistic EEFLs may also emerge from changes in predation regimes, as demonstrated by Gårdmark et al. (2003) using a theoretical model in which an age-structured population evolves in response to both harvesting and predation mortality. Harvesting reduces prey availability so that predator density decreases, thus

310 inducing relaxed predation and the associated natural selection for smaller body sizes. This result is likely to apply whenever predators of the harvested population directly select for smaller body sizes, 


\section{Size-dependent eco-evo feedback loops}

i.e., when predators preferentially prey on large-bodied individuals, on juveniles, or when they are non size-selective (Table 1).

315 A hallmark of eco-evolutionary dynamics is their tendency to remain cryptic if they are not anticipated and, hence, not specifically investigated (Kinnison et al. 2015). Size-dependent, antagonistic EEFLs are no exception, because the changes in natural selection oppose the effects of direct harvest selection and favour body-size stasis, an outcome that may erroneously be interpreted as direct harvest selection being too weak to drive any evolutionary response (e.g., Hilborn and Minte-Vera 2008). In fact,

320 however, body-size stasis of antagonistic EEFLs is associated with a fitness drop that may ultimately prevent evolutionary rescue (Fig. 2B). The fitness drop and resultant decreased population size may further jeopardize body-size evolvability (Box 1, Arrow $2 \rightarrow 3$ sequence in Fig. 1) which, together with a vanishing strength of selection due to a flat composite fitness landscape (Fig. 2B), decreases the probability for recovery. Overall, any situation in which harvesting is associated with body-size stasis

325 but severe population decline may be suspected to reflect an antagonistic EEFL.

\subsection{Synergistic EEFLS}

Synergistic EEFLs occur when the environmental feedback changes natural selection towards favouring smaller-than-initial body sizes in synergy with direct harvest selection (Fig. 2C). Synergistic

330 EEFLs may result, for instance, when harvesting, through reducing the density of large-sized individuals in the population, relaxes interference competition and cannibalism and associated natural selection for a large body size (Table 1). Recent experimental evidence in replicated fish populations suggests that this harvest-induced relaxation of interference competition and cannibalism can drive a rapid evolutionary divergence between harvested and non-harvested populations (Bouffet-Halle et al. 
335 2020). Synergistic EEFLs are also expected when predation favours larger body sizes and predators disappear due to harvest-induced prey shortage (Table 1, Jusufovski and Kuparinen 2020).

Qualitatively, the phenotypic outcome from synergistic EEFLs looks similar to the phenotypic outcome from EEFL-absent dynamics (Fig. 2A), though directional selection is stronger and expected trait variation faster. Synergistic EEFLs are thus likely to remain cryptic and to be interpreted as a large and rapid response to direct harvest-selection acting alone (e.g., Darimont et al. 2009). Compared to an EEFL-absent situation (Fig. 2A), however, synergistic EEFLs result in a magnified fitness peak on the composite fitness landscape (Fig. 2B) and, hence, favour larger population sizes at the body-size optimum and higher body-size evolvability (Arrow $2 \rightarrow 3$ sequence in Fig. 1). Hence, synergistic

345 EEFLs may favour evolutionary rescue and allow fast evolutionary rebound after relaxation of fishing. This is presumably the configuration that explains why pike, a highly cannibalistic species, showed a fast and large evolutionary response to varying harvesting intensity in Windermere (Edeline et al. 2007, Coltman 2008). Finally, synergistic EEFLs increase slope steepness around the fitness peak on the composite fitness landscape (Fig. 2C), resulting in stronger selection around and faster evolution towards the body-size optimum. Therefore, synergistic EEFLs are consistent with the observation that fishing-induced trait changes are often much faster than predicted by theoretical models that only assume direct harvest selection (Audzijonyte et al. 2013a).

These simple scenarios of antagonistic and synergistic EEFLs focus on the evolution of just one

355 harvested species alone. Fisheries, however, most often target not just one but several species within the ecological network, so that an ecosystem perspective on fishery management is required (White et al. 2012). Therefore, we now move to examining EEFLs when more than one species evolves. 


\section{Scenarios of harvest-induced EEFLs with multiple evolving species}

360 There is currently an emerging recognition that evolution in a given harvested species can induce coevolution in other species through changes in ecological interactions (Wood et al. 2018). However, understanding the evolutionary response to harvesting in a multispecific context is highly challenging (Audzijonyte et al. 2013b). Investigation on multispecies EEFLs requires to account simultaneously for the coevolution of the various body sizes, of the network structure, and to consider how one feeds back on the other (Loeuille and Loreau 2005). Direct data investigating the occurrence and magnitude of multispecies EEFLs are scarce. However, different empirical facts suggest that multispecies EEFLs may naturally emerge in exploited ecological networks.

First, empirical data suggest that predators are often larger than their prey in both terrestrial and aquatic systems (Cohen et al. 2003, Sinclair et al. 2003, Brose et al. 2006) and that predator-prey body-size ratios determine the strength of predation (Emmerson and Raffaelli 2004, Renneville et al. 2016). Therefore, we expect that selection on body size will change the distribution of interaction strengths, which largely constrains ecosystem functioning and stability (e.g., McCann et al. 1998, Rooney et al. 2006). Ultimately, rewiring and redistribution of interaction strengths may lead to extinctions in the network. For instance, evolution of larger body sizes can decrease the density of the evolving population thereby increasing its vulnerability to demographic stochasticity and potentially facilitating its extinction (evolutionary deterioration, see Glossary). At the same time, body-size evolution, by changing the strength of trophic interactions, may undermine predator or prey persistence through bottom-up or top-down constraints, respectively. Similarly, variations in interaction strengths will affect 
380 apparent competition (Holt et al. 1994), thereby changing coexistence conditions within the food web and possibly leading to competitive exclusions. Because the network structure, in turn, constrains the fitness of species within the community, multispecies EEFLs naturally emerge (Fig. 3).

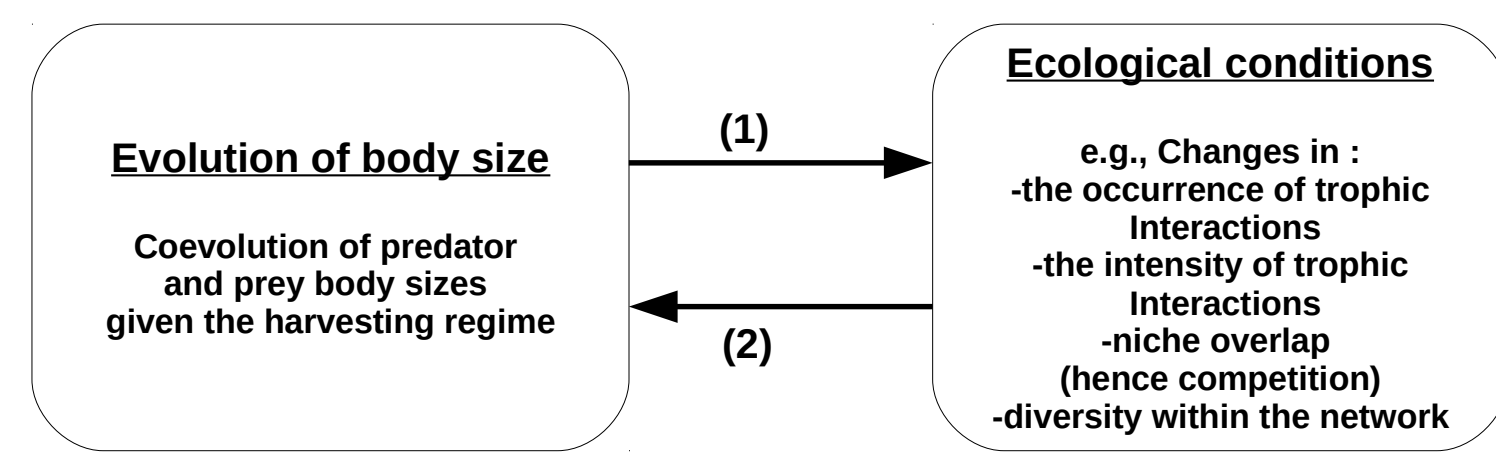

Figure 3: Components of multispecies EEFLs in food webs. (1) Coevolution of body sizes within the network under the new selective regime (harvesting) affects interaction patterns within the network, as well as coexistence conditions. (2) This new ecological context in turn changes the fitness of species (eg, due to changes in predation rates), thereby acting on body size coevolution.

Co-evolution strongly complicates EEFLs. In particular, the graphical framework from Fig. 2 no longer applies, because the environment now becomes multidimensional and evolution no longer optimizes fitness or population size of any given species (Meszéna et al. 2001, Metz et al. 2008). To keep our arguments as simple as possible we focus on a single co-evolving predator-prey pair, in which we examine two non-exclusive mechanisms for the emergence of EEFLs. We first examine the consequences of a "trophic relaxation", which occurs when decreased densities weaken the strength of the predator-prey link. Second, we examine the consequences of predators and prey having “asymmetric evolvability” for body size and, hence, evolving at a different pace in response to harvesting. For both mechanisms we consider that, before harvesting starts, the predator-prey pair resides at an evolutionary equilibrium. 


\subsection{Trophic relaxation}

Prey may be either smaller or larger than the preferred prey size of the predator. Prey sizes matching the preferred size are rarely expected, as prey may evolve away from such situations, but also because the distribution of body sizes does not usually maximize trophic interactions due to metabolic constraints, competition and the multiplicity of prey and predator species that also act as selective

Trophic relaxation

405

410

415

420

425
Asymmetric evolvability

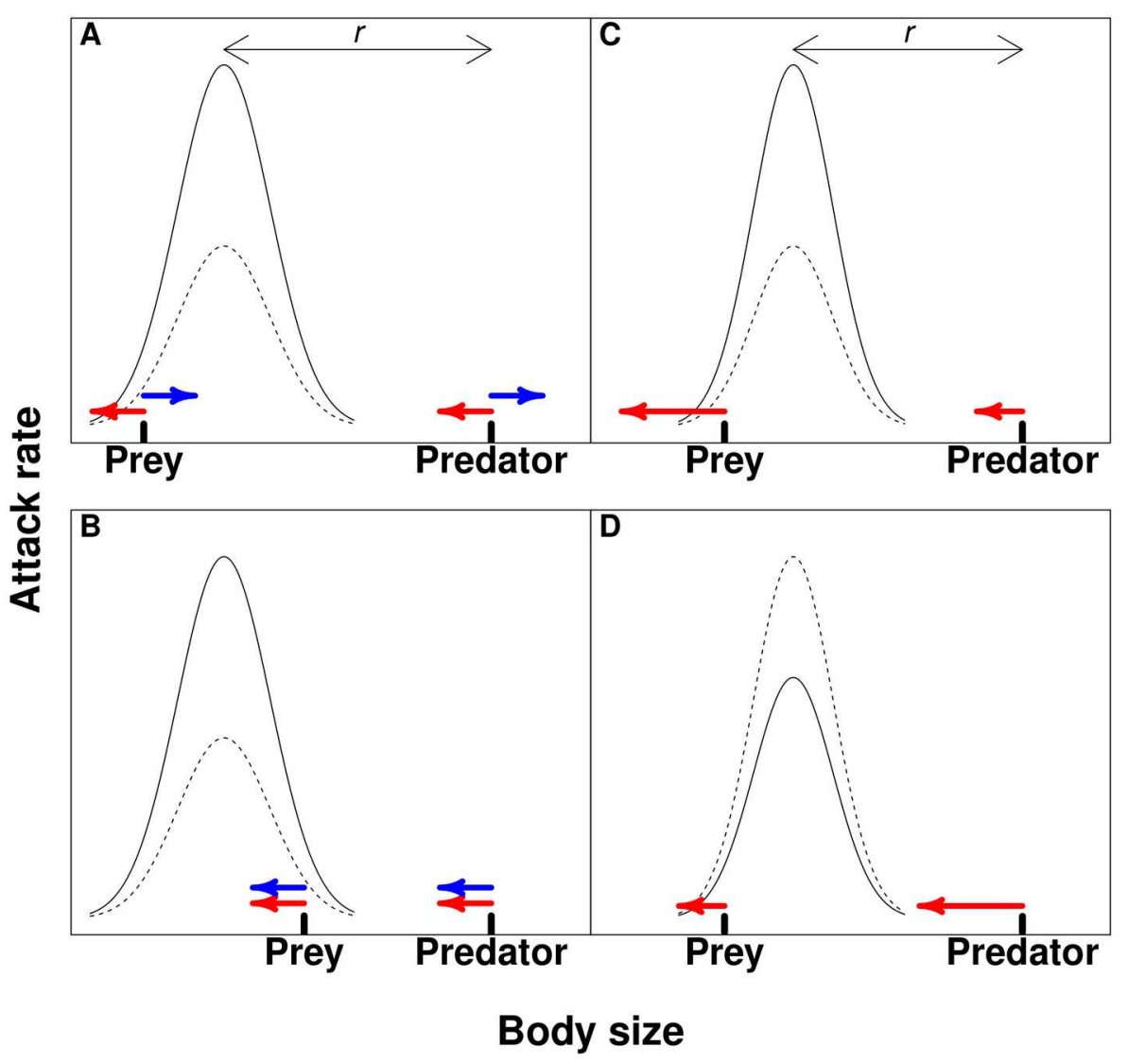

Figure 4. Alternative settings in coevolving predator-prey pairs. The Gaussian curves shows the predation intensity before (solid lines) and after (dashed lines) harvesting starts. Optimal prey size is set by the fixed ratio $r$. Red horizontal arrows show potential for body size evolution (i.e., body-size evolvability) in response to direct harvest selection. Blue arrows show natural-selection response to harvesting, i.e., the EEFL. A: Trophic relaxation leading to increased natural selection for a larger body size. B: Trophic relaxation leading to natural selection for a smaller body size. C: Body-size evolvability is larger in the prey than in the predator. D: Bodysize evolvability is larger in the predator than in the prey.

We now start harvesting both the prey and predator which,

hence, are both under direct harvest selection for a smaller body size (red arrows in Fig. 4). In Figs. 4A and $4 \mathrm{~B}$, red arrows have similar lengths indicating that both species evolve smaller body sizes at a 


\section{Size-dependent eco-evo feedback loops}

430 similar pace, such that no change is to be expected in their realized body-size ratio. However, because harvesting reduces population density in both the prey and predator, we expect a relaxation in the strength of the predator-prey link. This "trophic relaxation” is outlined in Figs. 4A and 4B by a decreased predation intensity (dotted Gaussian curves). Such a trophic relaxation may lead to opposite eco-evolutionary outcomes depending on whether initial prey size is smaller or larger than the

435 predator's optimal prey size.

In Fig. 4A, prey size is initially smaller than optimal for the predator, and the trophic relaxation thus results in relaxed natural selection for smaller body sizes in both the prey and predator (blue arrows). In other words, change in natural selection acts in opposition with direct harvest selection in an antagonistic EEFL. In case 4B, in contrast, prey size is initially larger than optimal predator size, and the trophic relaxation thus results in relaxed natural selection for larger body sizes, thus creating a synergistic EEFL. Of course, these relatively simple outcomes are complicated by feedbacks from intraspecific interactions (competition, cannibalism) that may either reinforce or oppose the effects of the predator-prey feedback (see Section 2).

\subsection{Asymmetric evolvability}

Assuming symmetric evolvability in the prey and predator (Figs. 4A and 4B) is likely unrealistic for most situations. Rather, body-size may be more evolvable in prey than predators (hence the longer red arrow in Fig. 4C), either because the trait is determined by different gene networks for the two species, or because the two species have very different population sizes, hence differing in accumulation of mutations or standing genetic variability. For instance, smaller (prey) body sizes are often associated with larger population numbers (Woodward et al. 2005). Under these settings, prey evolve smaller body 
sizes faster than their predator, move further away from predator's preferred prey size, and ultimately benefit from an evolution-induced trophic relaxation (Fig. 4C). The predator on the other hand, may become resource limited, so that further declines in predator population are expected. This is different from trophic relaxation in cases $4 \mathrm{~A}$ and $4 \mathrm{~B}$ which was the driver of evolution.

In Fig 4D, we sketch an opposite, perhaps less common situation in which predators have a higher body-size evolvability than their prey. This configuration may potentially result from prey being close

460 to a lower evolutionary limit for body size (Le Rouzic et al. 2020, Renneville et al. 2020). Under these settings, predators evolve smaller body sizes faster than prey, such that preferred prey size moves closer to prey size and a trophic magnification results. Such a coevolution therefore favours the maintenance of the trophic interaction. Note that these outcomes depend on prey being smaller than predator's preferred prey size in Figs. 4C and 4D, and are reversed when prey are larger than the 465 preferred prey size of the predator (i.e., trophic magnification in Fig. 4C and trophic relaxation Fig. 4D).

\subsection{More complex interaction networks}

In more complex networks, the multiplicity of trophic and non-trophic interactions may generate a

470 variety of counteracting selection gradients, so that evolution might be more constrained than in a single predator-prey link. If this hypothesis is true, EEFLs might well be more important in explaining evolutionary and ecological stasis rather than change (Ellner et al. 2011, Strauss 2014, Kinnison et al. 2015). Beyond very specific scenarios, network and eco-evolutionary complexities under harvesting scenarios are virtually impossible to grasp intuitively, and are even hard to handle through a 475 mathematical analysis. However, numerical simulations are certainly possible. In this regard, the 


\section{Size-dependent eco-evo feedback loops}

development of evolutionary models of food webs based on body size offer promising venues, as they already consider simultaneously evolution of body size and changes in the network structure (Loeuille and Loreau 2005, 2009, Brännström et al. 2011, Allhoff et al. 2015). Harvesting scenarios may be implemented in such models (Perälä and Kuparinen 2020), as has been done in other contexts (eg, 480 climate warming, Weinbach et al. 2017, Yacine et al. 2019).

\section{Management and perspectives}

So far, the vast majority of models used to project the eco-evolutionary consequences of fishing ignore natural selection on body size (but see Jusufovski and Kuparinen 2020). Hence, although quite

elaborated, these models are likely to underestimate either the demographic consequences of harvesting when antagonistic EEFLs are involved, or the rates of evolutionary change and recovery when synergistic EEFLs are involved. We recognize, however, that more empirical and experimental studies are needed to document the pathways, directions and strength of density-dependent selection acting on body size in harvested systems. In particular, it is important to document whether and when harvest-

490 induced EEFLs can be simplified into a one-dimensional, density-dependent process that can be handled by optimality approaches such as that outlined in Fig. 2. In Box 2, we provide an overview of the empirical methods currently available to progress in that direction.

\section{Box 2. Empirical exploration of size-dependent EEFLs: where to go next?}

495 Demonstrating a full selection-mediated EEFL requires showing both that natural selection drives evolutionary trait change and, in turn, that the resultant trait evolution alters the environment in such a way that natural selection acting back on the trait is modified (Figs. 1, 2). Considering also the 
evolvability-mediated pathways to EEFLs requires to further measure the effects of environmental changes on trait evolvability. Tackling such a complexity is challenging but, as we show below, not beyond of reach.

\section{Measuring natural selection and trait response to selection}

The form and strength of selection are most accurately measured by estimating fitness-traits relationships at the individual level (Arnold 2003) using, e.g., capture-recapture techniques. Alternatively, the directional component of selection may also be estimated from population and trait time series using the "Geber method", the age-structured price equation or integral projection models (Hairston et al. 2005, Ellner et al. 2011, van Benthem et al. 2017, Govaert 2018). A drawback of all these methods is that they measure selection acting on phenotypes, while evolution is concerned only by selection acting on the heritable component of phenotypes (Morrissey et al. 2010). To circumvent this problem, statistical approaches making use of the "animal model” (AM) of quantitative genetics were developed to specifically measure selection acting on the additive genetic component of traits and, hence, to accurately predict evolution (Hadfield 2008, Morrissey et al. 2010, Stinchcombe et al. 2014). AM-based approaches require pedigree data and are thus more readily implementable in small, closed systems than in large-scale fisheries (but see Koch et al. 2008).

Measuring the dependency of natural selection on the environment (Fig. 1, Arrow 1)

A pivotal condition for the emergence of selection-mediated EEFLs is that natural selection dynamically changes due to changes in the environment (Figs. 1, 2, Govaert et al. 2019). This may be checked a posteriori through measuring genotype-by-food interactions on body sizes. For instance, Bouffet-Halle et al. (2020) used this approach to show that harvest-induced evolution towards smaller 
Size-dependent eco-evo feedback loops

body sizes in experimental populations of medaka fish (Oryzias latipes) had evolved in a low-food but not in a high-food environment. This result suggested that medaka had evolved in response to densitydependent natural selection at high population density (low food), but not in response to direct harvest selection at low population density (high food). This approach, however, remains fragile because our understanding of genotype-by-food interactions remains limited, and other complementary results may be necessary to back-up conclusions from genotype-by-food analyses (Bouffet-Halle et al. 2020). When possible, selection-environment relationships should be measured directly using individual capture-recapture techniques (e.g., Haugen et al. 2007, Calsbeek and Smith 2007, Calsbeek and Cox 2010), keeping in mind the problems highlighted above of measuring selection at the phenotype level. Here also, these problems may be solved if the data permits applying the AM, which may be extended to estimate environment-selection relationships acting at the additive genetic level (Hunter et al. 2018).

Measuring the trait dependency of ecological dynamics (Fig. 1, Arrow 2)

Time series data may be used to quantify the feedback from phenotypic trait change to environmental variables. Since the inception of the Geber Method by Hairston et al. (2005) and Ellner et al. (2011), a multiplicity of more sophisticated methods have flourished. These methods are based either on inferring parameters for dynamic models from data (e.g., Rudy et al. 2017 and references therein), on non-parametric approaches such as Recurrent Neural Networks, or on hybrid approaches combining differential equations with neural networks (e.g., Bonnaffé et al. 2020 and references therein). Reviewing these methods is beyond the scope of this paper. 


\section{Size-dependent eco-evo feedback loops}

\section{Measuring the effects of the environment on trait evolvability (Fig. 1, Arrow 3)}

Evolvability may be measured using multiple metrics (e.g., Hansen et al. 2011, 2019), which condition approaches to exploring evolvability-mediated EEFLs. Here, we focused on additive genetic variance $\mathrm{V}_{\mathrm{A}}$ which is a commonly-used measure of evolvability (Box 1), and which we assumed to be positively linked to population size (see Reed and Frankham 2001 for a contrasted view). Estimation of $V_{A}$ relies on the $A M$, using either pedigrees or genetic markers of coancestry to construct relatedness matrices, with some caveats stressed by Lynch and Walsh (2018). The AM may further be extended to incorporate effects of environmental covariates on $\mathrm{V}_{\mathrm{A}}$ in so-called random regression approaches (Schaeffer 2004).

Importantly, our review suggests that the ecological and evolutionary consequences of harvesting will largely depend on the ecological factors that regulate the population and, hence, will likely be constrained by the details of the local network context. However, based on our above analysis we may still propose some general management rules accounting for size-dependent EEFLs. As highlighted by Engen et al. (2014), a very general consequence of density-dependent selection is that the more ecologically-sustainable strategies will also produce the less evolutionary changes. Therefore,

560 preventing population declines and alleviating evolutionary change are not independent lines of management but are instead highly intertwined management targets. If possible, management rules should further account for the probability of EEFLs to be either antagonistic or synergistic, because the former are far more detrimental than the later to population persistence and recovery and, hence, would impose lower exploitation rates. Ideally, an a priori knowledge of the direction of density-

565 dependent natural selection acting on body sizes could be gained using had hoc approaches (Box 2). Alternatively, a basic knowledge of the dominant ecological interactions could be used (Table 1). 
In co-evolving predator-prey pairs, managers may also account for body-size ratios and potential asymmetries in body-size evolvability, so as to classify their harvested system into one of the four

570 categories depicted in Fig. 4. Body-size ratios are well documented in the literature, and identification of a context prone to trophic relaxation or magnification should be relatively simple and lead to prudent exploitation. Prudent exploitation is also recommended if asymmetric body-size evolvability is suspected, especially when prey can escape predation (Fig. 4D), a situation in which exploitation rates should be stronger on the faster-evolving species so as to resorb asymmetry in evolvability. This

575 recommendation somehow converges towards "balanced harvesting”, a management approach based on spreading fishing mortality across the widest possible range of species and sizes in proportion to their natural productivity. Interestingly, such balanced strategies have already been advocated to conciliate yield and sustainability even in models that ignore evolution (Tromeur and Loeuille 2017). Although more research is clearly needed to test whether and under which conditions these general 580 recommendations hold true, we believe that far enough evidence is already available showing that a consideration of natural selection is highly needed if we are to improve our ability to accurately predict and manage the dynamics of harvested populations.

Acknowledgements: EE acknowledges financial support from the Norwegian Research Council 585 (projects EvoSize RCN 251307/F20 and REEF RCN 255601/E40) and from Rennes Métropole (project AIS 18C0356).

Conflict of interest disclosure: The authors of this article declare that they have no financial conflict of interest with the content of this article. NL is one of the PCIEcology recommenders. 
Size-dependent eco-evo feedback loops

\section{REFERENCES}

Abrams 2001. Modelling the adaptive dynamics of traits involved in inter- and intraspecific interactions: an assessment of three methods. - Ecol. Lett. 4: 166-175.

Abrams, P. A. and Rowe, L. 1996. The effects of predation on the age and size of maturity of prey. Evolution 50: 1052-1061.

Allhoff, K. T. et al. 2015. Evolutionary food web model based on body masses gives realistic networks with permanent species turnover. - Sci. Rep. 5: 10955.

Andersen, K. H. and Brander, K. 2009. Expected rate of fisheries-induced evolution is slow. - Proc. Natl. Acad. Sci. U. S. A. 106: 11657-11660.

Arendt, J. D. 1997. Adaptive intrinsic growth rates: an integration across taxa. - Q. Rev. Biol. 72: 149_ 177.

Arnold, S. J. 2003. Performance surfaces and adaptive landscapes. - Integr. Comp. Biol. 43: 367-375.

Audzijonyte, A. et al. 2013a. How fast is fisheries-induced evolution? Quantitative analysis of modelling and empirical studies. - Evol. Appl. 6: 585-595.

Audzijonyte, A. et al. 2013b. Ecological consequences of body size decline in harvested fish species: positive feedback loops in trophic interactions amplify human impact. - Biol. Lett. 9: 20121103.

Barneche, D. R. et al. 2018. Fish reproductive-energy output increases disproportionately with body size. - Science 360: 642.

Beckerman, A. P. et al. 2010. The reaction norm of size and age at maturity under multiple predator risk. - J. Anim. Ecol. 79: 1069-1076.

Biro, P. A. and Post, J. R. 2008. Rapid depletion of genotypes with fast growth and bold personality traits from harvested fish populations. - Proc. Natl. Acad. Sci. U. S. A. 105: 2919-2922.

Bonnaffé, W. et al. 2020. Neural ordinary differential equations for ecological and evolutionary time series analysis. - Authorea in press.

Borrell, B. 2013. Ocean conservation: a big fight over little fish. - Nature 493: 597-598.

Bouffet-Halle, A. et al. 2020. Density-dependent selection mediates harvest-induced trait changes. Ecol. Lett. in press.

Brännström, Å. et al. 2011. Emergence and maintenance of biodiversity in an evolutionary food-web model. - Theor. Ecol. 4: 467-478. 
Size-dependent eco-evo feedback loops

Brooks, J. L. and Dodson, S. I. 1965. Predation, body size, and composition of plankton. - Science 150: 28.

Brose, U. et al. 2006. Consumer-resource body-size relationships in natural food webs. - Ecology 87: 2411-2417.

Brown, J. H. et al. 2004. Toward a metabolic theory of ecology. - Ecology 85: 1771-1789.

Calsbeek, R. and Smith, T. B. 2007. Probing the adaptive landscape using experimental islands: density-dependent natural selection on lizard body size. - Evolution 61: 1052-1061.

Calsbeek, R. and Cox, R. M. 2010. Experimentally assessing the relative importance of predation and competition as agents of selection. - Nature 465: 613.

Carlson, S. M. et al. 2007. Four decades of opposing natural and human-induced artificial selection acting on Windermere pike (Esox lucius). - Ecol. Lett. 10: 512-521.

Carlson, S. M. et al. 2014. Evolutionary rescue in a changing world. - Trends Ecol. Evol. 29: 521-530.

Carroll, S. P. et al. 2007. Evolution on ecological time-scales. - Funct. Ecol. 21: 387-393.

Chase, J. M. 1999. To grow or to reproduce? The role of life-history plasticity in food web dynamics. Am. Nat. 154: 571-586.

Claessen, D. et al. 2002. The impact of size-dependent predation on population dynamics and individual life history. - Ecology 83: 1660-1675.

Claessen, D. et al. 2004. Population dynamic theory of size-dependent cannibalism. - Proc. R. Soc. Lond. B Biol. Sci. 271: 333.

Cohen, J. E. et al. 2003. Ecological community description using the food web, species abundance, and body size. - Proc. Natl. Acad. Sci. U. S. A. 100: 1781-1786.

Conover, D. O. and Munch, S. B. 2002. Sustaining fisheries yields over evolutionary time scales. Science 297: 94-96.

Crow, J. F. 2008. Maintaining evolvability. - J. Genet. 87: 349-353.

Darimont, C. T. et al. 2009. Human predators outpace other agents of trait change in the wild. - Proc. Natl. Acad. Sci. U. S. A. 106: 952-954.

Day, T. et al. 2002. The role of size-specific predation in the evolution and diversification of prey life histories. - Evolution 56: 877-887.

De Roos, A. M. et al. 2003. The influence of size-dependent life-history traits on the structure and dynamics of populations and communities. - Ecol. Lett. 6: 473-487. 
Devine, J. A. and Heino, M. 2011. Investigating the drivers of maturation dynamics in Barents Sea haddock (Melanogrammus aeglefinus). - Fish. Res. 110: 441-449.

Dieckmann, U. and Law, R. 1996. The dynamical theory of coevolution: a derivation from stochastic ecological processes. - J. Math. Biol. 34: 579-612.

Edeline, E. et al. 2007. Trait changes in a harvested population are driven by a dynamic tug-of-war between natural and harvest selection. - Proc. Natl. Acad. Sci. U. S. A. 104: 15799-15804.

Edeline, E. et al. 2009. Harvest-induced disruptive selection increases variance in fitness-related traits. - Proc. R. Soc. Lond. B Biol. Sci. 276: 4163-4171.

Edeline, E. et al. 2016. Empirical evidence for competition-driven semelparity in wild medaka. - Popul. Ecol. 58: 371-383.

Edley, M. T. and Law, R. 1988. Evolution of life histories and yields in experimental populations of Daphnia magna. - Biol. J. Linn. Soc. 34: 309-326.

Ellner, S. P. et al. 2011. Does rapid evolution matter? Measuring the rate of contemporary evolution and its impacts on ecological dynamics. - Ecol. Lett. 14: 603-614.

Emmerson, M. C. and Raffaelli, D. 2004. Predator-prey body size, interaction strength and the stability of a real food web. - J. Anim. Ecol. 73: 399-409.

Engen, S. et al. 2014. Evolutionary consequences of nonselective harvesting in density-dependent populations. - Am. Nat. 184: 714-726.

Ernande, B. et al. 2004. Adaptive changes in harvested populations: plasticity and evolution of age and size at maturation. - Proc. Biol. Sci. 271: 415-423.

Fenberg, P. B. and Roy, K. 2008. Ecological and evolutionary consequences of size-selective harvesting: how much do we know? - Mol. Ecol. 17: 209-220.

Fleming, I. A. and Gross, M. R. 1994. Breeding competition in a Pacific salmon (coho: Oncorhynchus kisutch): measures of natural and sexual selection. - Evolution 48: 637-657.

Fox, L. R. 1975. Cannibalism in natural populations. - Annu. Rev. Ecol. Syst. 6: 87-106.

Gadgil, M. and Bossert, W. H. 1970. Life historical consequences of natural selection. - Am. Nat. 104: $1-24$.

Gårdmark, A. and Dieckmann, U. 2006. Disparate maturation adaptations to size-dependent mortality. Proc. R. Soc. Lond. B Biol. Sci. 273: 2185-2192.

Gårdmark, A. et al. 2003. Life-history evolution in harvested populations: the role of natural predation. - Evol. Ecol. Res. 5: 239-257. 


\section{Size-dependent eco-evo feedback loops}

Gomulkiewicz, R. and Holt, R. D. 1995. When does evolution by natural selection prevent extinction? Evolution 49: 201-207.

Govaert, L. 2018. Eco-evolutionary partitioning metrics: a practical guide for biologists. - Belg. J. Zool. 148: 167-202.

Govaert, L. et al. 2019. Eco-evolutionary feedbacks - Theoretical models and perspectives. - Funct. Ecol. 33: 13-30.

Grant, P. R. and Grant, B. R. 2002. Unpredictable evolution in a 30-year study of Darwin's finches. Science 296: 707-711.

Hadfield, J. D. 2008. Estimating evolutionary parameters when viability selection is operating. - Proc. R. Soc. B Biol. Sci. 275: 723-734.

Hairston, N. G. et al. 2005. Rapid evolution and the convergence of ecological and evolutionary time. Ecol. Lett. 8: 1114-1127.

Hamrin, S. F. and Persson, L. 1986. Asymmetrical competition between age classes as a factor causing population oscillations in an obligate planktivorous fish species. - Oikos 47: 223-232.

Hansen, ThomasF. et al. 2011. Heritability is not evolvability. - Evol. Biol. 38: 258-277.

Hansen, T. F. et al. 2019. Predicting evolutionary potential: a numerical test of evolvability measures. Evolution 73: 689-703.

Haugen, T. O. et al. 2007. Density dependence and density independence in the demography and dispersal of pike over four decades. - Ecol. Monogr. 77: 483-502.

Heino, M. et al. 2013. Can fisheries-induced evolution shift reference points for fisheries management? - ICES J. Mar. Sci. J. Cons. 70: 707-721.

Heino, M. et al. 2015. Fisheries-induced evolution. - Annu. Rev. Ecol. Evol. Syst. 46: 461-480.

Hilborn, R. and Walters, C. 1992. Quantitative fisheries stock assessment: choice, dynamics and uncertainty. - Springer US.

Hilborn, R. and Minte-Vera, C. V. 2008. Fisheries-induced changes in growth rates in marine fisheries: are they significant? - Bull. Mar. Sci. 83: 95-105.

Holliday, R. 1989. Food, reproduction and longevity: is the extended lifespan of calorie-restricted animals an evolutionary adaptation? - BioEssays News Rev. Mol. Cell. Dev. Biol. 10: 125-127.

Holt, R. D. et al. 1994. Simple rules for interspecific dominance in systems with exploitative and apparent competition. - Am. Nat. 144: 741-771. 
Hunter, D. C. et al. 2018. Quantification and decomposition of environment-selection relationships. Evolution 72: 851-866.

Jusufovski, D. and Kuparinen, A. 2020. Exploring individual and population eco-evolutionary feedbacks under the coupled effects of fishing and predation. - Fish. Res. 231: 105713.

Kingsolver, J. G. and Pfennig, D. W. 2004. Individual-level selection as a cause of Cope's rule of phyletic size increase. - Evolution 58: 1608-1612.

Kinnison, M. T. et al. 2015. Cryptic eco-evolutionary dynamics. - Ann. N. Y. Acad. Sci. 1360: 120-144.

Koch, M. et al. 2008. Pedigree reconstruction in wild cichlid fish populations. - Mol. Ecol. 17: 4500_ 4511.

Kooijman, S. A. L. M. 2010. Dynamic energy budget theory for metabolic organisation. - Cambridge University Press.

Kristiansen, T. S. and Svåsand, T. 1998. Effect of size-selective mortality on growth of coastal cod illustrated by tagging data and an individual-based growth and mortality model. - J. Fish Biol. 52: 688-705.

Kuparinen, A. and Merilä, J. 2007. Detecting and managing fisheries-induced evolution. - Trends Ecol. Evol. 22: 652-659.

Kuparinen, A. and Hutchings, J. A. 2017. Genetic architecture of age at maturity can generate divergent and disruptive harvest-induced evolution. - Philos. Trans. R. Soc. B Biol. Sci. 372: 20160035.

Lande, R. and Arnold, S. J. 1983. The measurement of selection on correlated characters. - Evolution 37: 1210-1226.

Law, R. 2000. Fishing, selection, and phenotypic evolution. - ICES J. Mar. Sci. J. Cons. 57: 659-668.

Le Bourlot, V. et al. 2014. Interference versus exploitative competition in the regulation of sizestructured populations. - Am. Nat. 184: 609-623.

Le Rouzic, A. et al. 2020. Unidirectional response to bidirectional selection on body size. II Quantitative genetics. - Ecol. Evol. 10: 11453-11466.

Loeuille, N. and Loreau, M. 2005. Evolutionary emergence of size-structured food webs. - Proc. Natl. Acad. Sci. U. S. A. 102: 5761-5766.

Loeuille, N. and Loreau, M. 2006. Evolution of body size in food webs: does the energetic equivalence rule hold? - Ecol. Lett. 9: 171-178.

Loeuille, N. and Loreau, M. 2009. Emergence of complex food web structure in community evolution models. - In: Verhoef, H. A. and Morin, P. J. (eds), Community ecology: processes, models, and applications. 1st ed.n. Oxford University Press, pp. 163-178. 


\section{Size-dependent eco-evo feedback loops}

Lynch, M. and Walsh, B. 2018. Evolution and selection of quantitative traits. - Oxford University Press.

Marty, L. et al. 2014. Temporal trends in age and size at maturation of four North Sea gadid species: cod, haddock, whiting and Norway pout. - Mar. Ecol. Prog. Ser. 497: 179-197.

Marty, L. et al. 2015. Fisheries-induced neutral and adaptive evolution in exploited fish populations and consequences for their adaptive potential. - Evol. Appl. 8: 47-63.

McCann, K. et al. 1998. Weak trophic interactions and the balance of nature. - Nature 395: 794.

Meszéna, G. et al. 2001. Evolutionary optimisation models and matrix games in the unified perspective of adaptive dynamics. - Selection 2: 193-220.

Metz, J. A. J. et al. 2008. When does evolution optimize? - Evol. Ecol. Res. 10: 629-654.

Morrissey, M. B. et al. 2010. The danger of applying the breeder's equation in observational studies of natural populations. - J. Evol. Biol. 23: 2277-2288.

Olsen, E. M. and Moland, E. 2011. Fitness landscape of Atlantic cod shaped by harvest selection and natural selection. - Evol. Ecol. 25: 695-710.

Orr, H. A. 2007. Absolute fitness, relative fitness, and utility. - Evolution 61: 2997-3000.

Perälä, T. and Kuparinen, A. 2020. Eco-evolutionary dynamics driven by fishing: from single species models to dynamic evolution within complex food webs. - Evol. Appl. in press.

Perez, K. O. and Munch, S. B. 2010. Extreme selection on size in the early lives of fish. - Evolution 64: 2450-2457.

Persson, L. 1985. Asymmetrical competition: are larger animals competitively superior? - Am. Nat. 126: 261-266.

Persson, L. and De Roos, A. M. 2006. Food-dependent individual growth and population dynamics in fishes. - J. Fish Biol. 69: 1-20.

Persson, L. et al. 1998. Ontogenetic scaling of foraging rates and the dynamics of a size-structured consumer-resource model. - Theor. Popul. Biol. 54: 270-293.

Peters, R. H. 1983. The ecological implications of body size. - Cambridge University Press.

Post, J. R. et al. 1999. Density-dependent processes in structured fish populations: interaction strengths in whole-lake experiments. - Ecol. Monogr. 69: 155-175.

Reed, D. H. and Frankham, R. 2001. How closely correlated are molecular and quantitative measures of genetic variation? A meta-analysis. - Evolution 55: 1095-1103.

Renneville, C. et al. 2016. Morphological drivers of trophic cascades. - Oikos 125: 1193-1202. 
Renneville, C. et al. 2020. Unidirectional response to bidirectional selection on body size. I. Phenotypic, life-history, and endocrine responses. - Ecol. Evol. 10: 10571-10592.

Roff, D. A. 1992. The evolution of life histories. - Chapman \& Hall.

Rooney, N. et al. 2006. Structural asymmetry and the stability of diverse food webs. - Nature 442: 265269.

Rudy, S. H. et al. 2017. Data-driven discovery of partial differential equations. - Sci. Adv. 3: e1602614.

Schaefer, M. B. 1954. Some aspects of the dynamics of populations important to the management of commercial marine fisheries. - Inter-Am. Trop. Tuna Comm. Bull. 1: 23-56.

Schaeffer, L. R. 2004. Application of random regression models in animal breeding. - Livest. Prod. Sci. 86: 35-45.

Sgrò, C. M. and Partridge, L. 2000. Evolutionary responses of the life history of wild-caught Drosophila melanogaster to two standard methods of laboratory culture. - Am. Nat. 156: 341353.

Silva, A. et al. 2013. Long-term changes in maturation of sardine, Sardina pilchardus, in Portuguese waters. - Sci. Mar. 77: 429-438.

Sinclair, A. R. E. et al. 2003. Patterns of predation in a diverse predator-prey system. - Nature 425: 288-290.

Stige, L. C. et al. 2019. Density- and size-dependent mortality in fish early life stages. - Fish Fish. 20: 962-976.

Stinchcombe, J. R. et al. 2014. Estimating uncertainty in multivariate responses to selection. Evolution 68: 1188-1196.

Stockwell, C. A. et al. 2003. Contemporary evolution meets conservation biology. - Trends Ecol. Evol. 18: 94-101.

Strauss, S. Y. 2014. Ecological and evolutionary responses in complex communities: implications for invasions and eco-evolutionary feedbacks. - Oikos 123: 257-266.

Swain, D. P. et al. 2007. Evolutionary response to size-selective mortality in an exploited fish population. - Proc. R. Soc. B Biol. Sci. 274: 1015.

Taylor, B. E. and Gabriel, W. 1992. To grow or not to grow: optimal resource allocation for Daphnia. Am. Nat. 139: 248-266.

Tilman, D. 1982. Resource competition and community structure. - Princeton University Press.

Trippel, E. A. 1995. Age at maturity as a stress indicator in fisheries. - BioScience 45: 759-771. 
Tromeur, E. and Loeuille, N. 2017. Balancing yield with resilience and conservation objectives in harvested predator-prey communities. - Oikos 126: 1780-1789.

van Benthem, K. J. et al. 2017. Disentangling evolutionary, plastic and demographic processes underlying trait dynamics: a review of four frameworks. - Methods Ecol. Evol. 8: 75-85.

van de Wolfshaar, K. E. et al. 2008. Population feedback after successful invasion leads to ecological suicide in seasonal environments. - Ecology 89: 259-268.

Verhulst, P. F. 1838. Notice sur la loi que la population suit dans son accroissement. - Corresp. Mathématique Phys. 10: 113-121.

Walsh, M. R. et al. 2006. Maladaptive changes in multiple traits caused by fishing: impediments to population recovery. - Ecol. Lett. 9: 142-148.

Weinbach, A. et al. 2017. Selective effects of temperature on body mass depend on trophic interactions and network position. - bioRxiv: 233742.

Wellborn, G. A. 1994. Size-biased predation and prey life histories: a comparative study of freshwater amphipod populations. - Ecology 75: 2104-2117.

White, C. et al. 2012. The value of coordinated management of interacting ecosystem services. - Ecol. Lett. 15: 509-519.

Wood, Z. T. et al. 2018. Eco-evolutionary feedbacks from non-target species influence harvest yield and sustainability. - Sci. Rep. 8: 6389.

Woodward, G. et al. 2005. Body size in ecological networks. - Trends Ecol. Evol. 20: 402-409. 